\title{
2020 Proceedings
}

\author{
The Boro Kimono \\ Emily Krull and Young-A Lee (Design Mentor) \\ Auburn University, USA
}

Keywords: Sustainability, zero waste fashion, denim, hand-stitching

Design Mentor Statement. This work was completed as part of the upcycling denim design challenge that was announced to all eligible design students within the department to enhance their sustainability awareness. For this challenge, the student was tasked with creating a design using the minimum of $80 \%$ recycled denim applying zero waste design approach. The student had to demonstrate understanding and ability in both the conceptual and technical aspects of zero waste design by developing the concept, selecting materials, creating patterns, and producing the garment. This garment represents delicate craftsmanship blended with a personal style and aesthetic. This particular student's work is of extremely high quality. It has a strong conceptual goal, which has been met through successful integration of the upcycling design intention with (a) the use of recycled denim, (b) application of culture-embedded hand stitching technique, and (c) consideration of the design's transformability or multi-functionality. This patchwork design using the Sashiko stitch inspired by the Japanese traditional Boro mending technique is aesthetically pleasing along with delivering functional and expressive desires. This design is cohesive and innovative throughout the design concept, design, and the construction techniques. All aspects of the garment construction are professionally executed.

Statement of Purpose. The Boro Kimono, showcasing the concept of zero waste fashion, was designed as

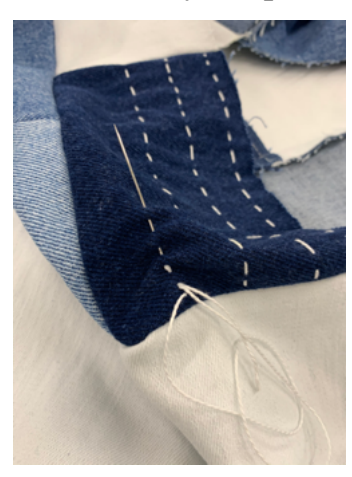

a single look for the upcycling denim design challenge initiated by the department. Challenged to create a single garment consisting of $80 \%$ recycled denim with the remaining fabric made of $100 \%$ cotton materials, this kimono jacket embodies versatility and sustainability. The inspiration behind this design came from the traditional Japanese Boro mending technique used to repurpose fabrics that were saved within a family over time and eventually sewn into a patchwork garment (Green, 2017). The Sashiko stitch was a main addition to each Boro piece, a variety of small running stitches that brought a purposeful yet decorative value to each garment. This denim kimono consists of an outer jacket made completely out of recycled denim jeans with a $100 \%$ cotton lining made from recycled button-down dress shirts.

Aesthetic Properties and Visual Impact. This denim look embodies versatility and sustainability through its $100 \%$ recycled design. Different tones of denim jean fabric create the patchwork design found throughout the kimono, complimenting the Sashiko stitch details delicately placed throughout the garment. Drawing inspiration from the Japanese traditional Boro mending and its versatility, this look can be transformed into a shorter kimono jacket or vest with a removable tiled panel and detachable kimono sleeves. The Boro Kimono has two other detachable design elements, a denim belt and a large pocket attached to the inner lining, both designed with the zero waste concept in mind. This garment was

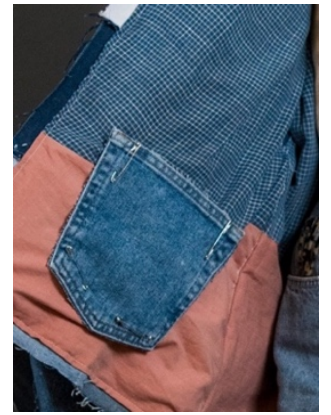

Page 1 of 3

(c) 2020 The author(s). Published under a Creative Commons Attribution License

(https://creativecommons.org/licenses/by/4.0/), which permits unrestricted use, distribution, and reproduction in any medium, provided the original work is properly cited.

ITAA Proceedings, \#77 - https: //itaaonline.org 
produced with intricate hand-stitching and patchwork detailing with the intention to be worn as a durable and stylish addition to one's wardrobe.

Process, Technique, and Execution. The design and development of The Boro Kimono includes multiple steps: design ideation, material selection, pattern drafting and development, prototype production, model

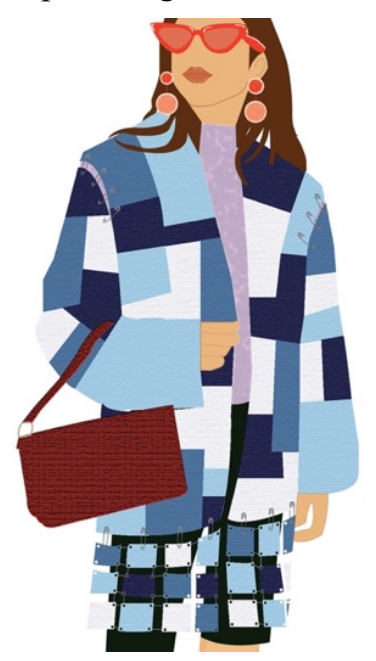

fitting, and the assembly of the final design. To begin this process, I conducted extensive research on the history of Boro mending and traditional Sashiko hand-stitching. Upon sketching out my garment silhouette and the transformable design elements, I specifically selected the recycled denim material used from local thrift stores, friends, and family. This step added a personal touch to the kimono that was commonly included in traditional Japanese Boro garments. When assembling the final garment, each individual denim piece was carefully tailored to each pattern piece to achieve the patchwork design on the outer jacket, and the same process was conducted for the inner cotton lining. The intricate craftsmanship of this design adds an elevated quality to this look and is showcased through the tiled denim panel with individual hand-punched grommets and the traditional Sashiko technique that was hand-stitched on several patches throughout the design. These detachable elements enhance the multi-functionality of this kimono and add a new level of innovation and personalization to sustainable fashion.

Cohesion. The cohesiveness of The Boro Kimono is conveyed through the use of $100 \%$ recycled denim and cotton materials throughout the entirety of this garment. The patchwork denim outer jacket and patchwork cotton lining bring together the traditional Japanese influences and techniques, while the addition of elements such as silver safety pins and grommets add a level of modernity and innovation to the design. Sustainable fashion and Boro mending both centralize around the concept of repurposing old materials while bringing a new garment to life containing history and intricate craftsmanship, and this denim kimono showcases all of these elements.

Originality and Innovation. As sustainable fashion is becoming the new "normal" within the apparel industry, I wanted to create a design that married traditional technique and style with innovation. The Boro Kimono encompasses the elements of historical Boro mending and is elevated with unique transformable elements. Unlike any garment I have produced in the past, this piece was made entirely with recycled materials and can be worn in many different styles ranging from a longer kimono to a shorter kimono vest. Although this design includes several hand-crafted techniques, it is suitable for every day wear while also remaining environmentally conscious. This denim kimono remains true to its traditional Japanese inspiration, but stands as a high quality piece derived from the concept of zero waste fashion.

Measurements: Female medium (size 6-8) - 34"x26"x35" (bust x waist x hip)

Date Completed: December 4, 2019

Page 2 of 3

(c) 2020 The author(s). Published under a Creative Commons Attribution License

(https://creativecommons.org/licenses/by/4.0/), which permits unrestricted use, distribution, and reproduction

in any medium, provided the original work is properly cited.

ITAA Proceedings, \#77 - https://itaaonline.org 


\section{References}

Green, M. (2017, September). Authentic Japanese boro, boro mending, and boro-inspired patchwork. https://www.sewingmachinesplus.com/sewblog/authentic-japanese-boro-boro-mending-boroinspired-patchwork/ 


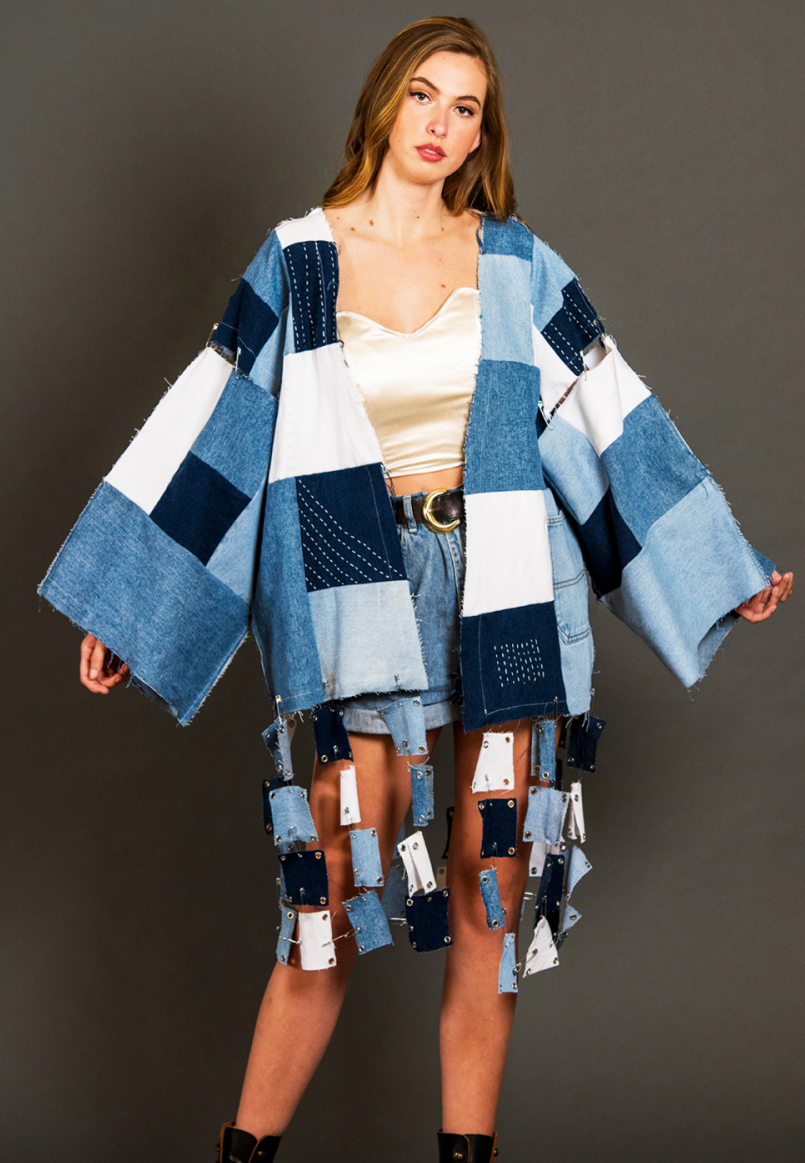

Image A Front

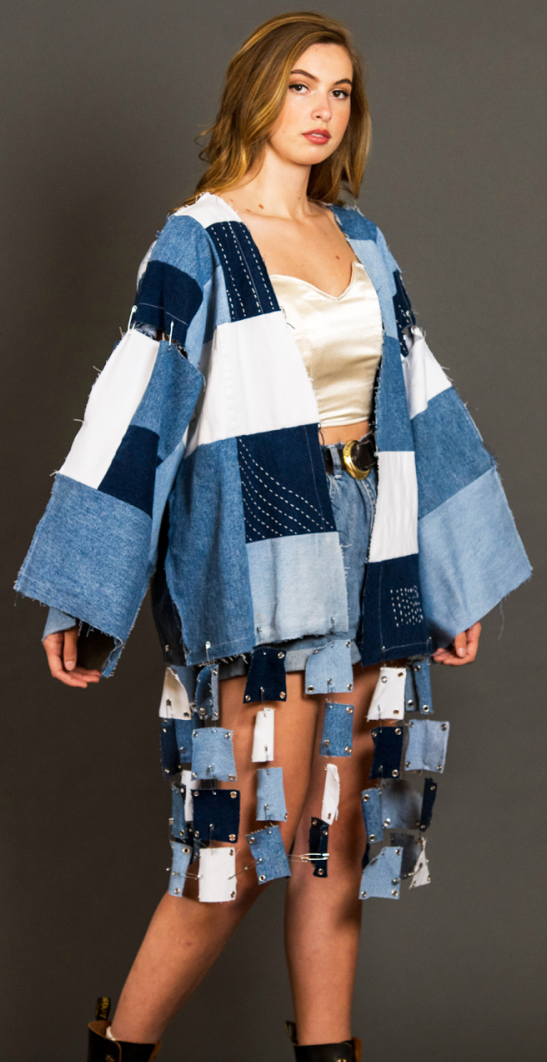

Image C Profile

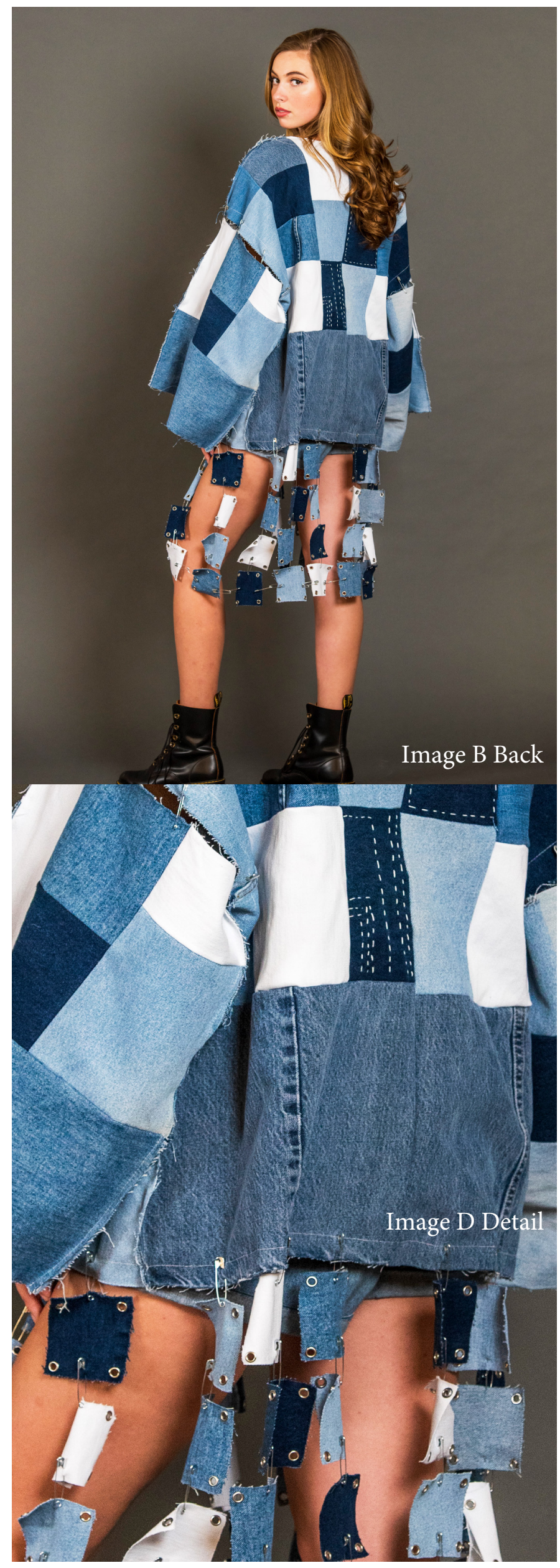

